\title{
The Potential Markers of Circulating microRNAs and long non-coding RNAs in Alzheimer's Disease
}

\author{
Yanfang Zhao $^{1 *}$, Yuan Zhang ${ }^{2}$, Lei Zhang ${ }^{2}$, Yanhan Dong ${ }^{2}$, Hongfang Ji ${ }^{1}$, Liang Shen ${ }^{1}$ \\ ${ }^{1}$ Institute of Biomedical Research, Shandong Provincial Research Center for Bioinformatic Engineering and \\ Technique, Zibo Key Laboratory of New Drug Development of Neurodegenerative diseases, School for Life \\ Science, Shandong University of Technology, Zibo, China. \\ ${ }^{2}$ Institute for Translational Medicine, Qingdao University, Qingdao, China.
}

[Received August 23, 2018; Revised November 5, 2018; Accepted November 5, 2018]

\begin{abstract}
Alzheimer's disease (AD) is a neurodegenerative disorder and one of the leading causes of disability and mortality in the late life with no curative treatment currently. Thus, it is urgently to establish sensitive and non-invasive biomarkers for AD diagnosis, particularly in the early stage. Recently, emerging number of microRNAs (miRNAs) and long-noncoding RNAs (IncRNAs) are considered as effective biomarkers in various diseases as they possess characteristics of stable, resistant to RNAase digestion and many extreme conditions in circulatory fluid. This review highlights recent advances in the identification of the aberrantly expressed miRNAs and IncRNAs in circulatory network for detection of AD. We summarized the abnormal expressed miRNAs in blood and cerebrospinal fluid (CSF), and detailed discussed the functions and molecular mechanism of serum or plasma miRNAs-miR-195, miR-155, miR-34a, miR-9, miR-206, miR-125b and miR-29 in the regulation of AD progression. In addition, we also elaborated the role of circulating IncRNA major including beta-site APP cleaving enzyme 1 (BACE1) and its antisense IncRNA BACE1-AS in AD pathological advancement. In brief, confirming the aberrantly expressed circulating miRNAs and IncRNAs will provide an effective testing tools for treatment of AD in the future.
\end{abstract}

Key words: Alzheimer's disease, circulating, miRNA, lncRNA

\begin{abstract}
Alzheimer's disease (AD) is one of the most prevalent age-related neurodegenerative disorders and a leading cause of disability and mortality in the late life [1]. It is characterized by the pathological changes including the formation of intracellular neurofibrillary tangles, accumulation of amyloid- $\beta$ (A $\beta$ ) peptides and Tau proteins, which lead to variable emotion alteration, personality changes, inappropriate social behaviors, progressive memory impairment and cognitive deficits, ultimately cause death [1-5]. With the accelerated process of aging society, aging related diseases especially $\mathrm{AD}$
\end{abstract}

bring a serious public health challenge. Six to eight in ten dementia patients have a diagnosis of AD [6]. An estimated 50 million people suffer from $\mathrm{AD}$ worldwide currently [6]. More seriously, the incidence of $\mathrm{AD}$ throughout the world is predicted to be 75.6 million by 2030 and 135.5 million by 2050 , which is almost triple the existing population affected [7].

The cause and molecular mechanism of $\mathrm{AD}$ pathogenesis remain largely unclear due to $\mathrm{AD}$ is one of the most complicated and complex age-related disease. Recently, growing advancement has made in diagnosis

*Correspondence should be addressed to: Dr. Yanfang Zhao, Institute of Biomedical Research, School for Life Science, Shandong University of Technology, Zibo, China. E-mail: zhaoyanfang1@126.com

Copyright: () 2018 Zhao Y et al. This is an open-access article distributed under the terms of the Creative Commons Attribution License, which permits unrestricted use, distribution, and reproduction in any medium, provided the original author and source are credited. 
and pharmacotherapy of $\mathrm{AD}$, however, no effective cure and prevention measure is able to halt the disease development or reverse the brain alteration. The diagnosis of the disease is based on the history of symptoms, thus, reliable biomarker for early diagnosis is extremely pivotal for prevention the $\mathrm{AD}$ process. In this review, we summarized the correlation between the ectopic expressed microRNAs (miRNAs), long noncoding RNAs (lncRNAs) in circulatory fluid and AD patients (Fig. 1), which providing competent evidence for searching novel therapy targets and biomarkers for AD.

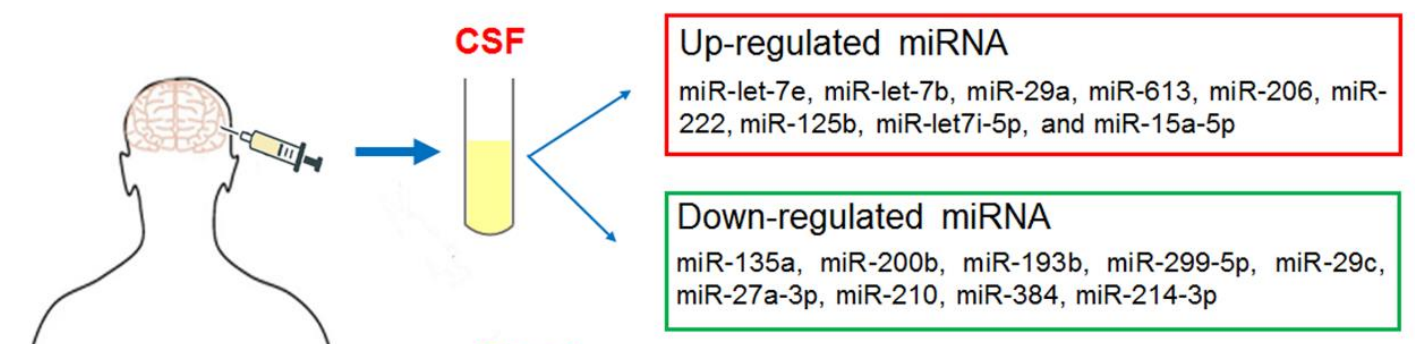

Blood

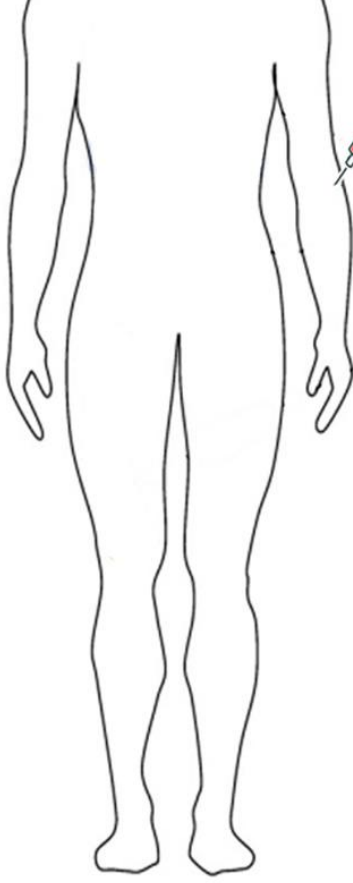

\section{AD pathological condition}

Figure 1. Circulating biomarkers in AD pathological condition. Plasma/serum biomarkers including miRNAs and lncRNAs, and cerebrospinal fluid (CSF) miRNAs.

\section{Circulating miRNA and AD}

\section{Circulating miRNA}

miRNAs are a family of short non-coding RNAs with approximately 18 22 nucleotides, which suppress protein expression by binding the 3'untranslated region (3'UTR) of protein mRNA or promoting mRNA degradation [8]. Not surprisingly, miRNAs related pathways participate in various diseases including neurodegenerative diseases [9]. As one of major great challenges in biomarker analysis in central nervous system diseases, it is urgently to search a suitable, non-invasive and blood-based early biomarker for AD. Recently, circulating miRNAs have attracted more attention with several advantages to be good biomarkers. They are stable in the circulation, resistant to RNAase digestion and many extreme conditions including extreme $\mathrm{pH}$, high temperature, extended storage, and multiple freeze-thaw cycles [10]. More importantly, miRNAs were able to be detected in all of the 12 body fluids [11], and the differential expression levels are tightly involved in various diseases or certain biological/pathological stages [12]. Here, blood and cerebrospinal fluid (CSF) miRNAs are introduced in this review as potential biomarkers in $\mathrm{AD}$ diagnosis. 


\section{Blood miRNA and $A D$}

It is a simple, affordable, less invasive or time saving detection method to explore the circulatory miRNAs in the blood as large-scale peripheral markers of patients with AD. A quantity of studies elaborated that the expression levels of miRNAs were checked in the blood of $\mathrm{AD}$ patients and normal controls at the same age (Fig. 1). The expression levels of miRNAs including miR-135a [13], miR-384 [13], miR-455-3p [14], miR-4668-5p [14], miR-146a-5p, miR-106b-3p, miR-195-5p, miR-20b-5p, and miR-497-5p [15], hsa-miR-101, hsa-miR-155, hasmiR-9 [16], miR-206 [17], miR-146a-5p, miR-106b-3p, miR-195-5p, miR-20b-5p, miR-497-5p [15], miR-519 [18], miR-200c [19], miR-590-5p and miR-142-5p [20] were up-regulated, while miR-193b [13], has-miR-501-3p [21], miR-125b-3p, miR-29c-3p, miR-93-5p, miR-19b-3p [15], hsa-let-7d-5p, hsa-let-7g-5p, hsa-miR-15b, hasmiR-191-5p, has-miR-26b-5p, hsa-miR-29b, hsa-miR342-3p [16], miR-135b [22], miR-29, miR-125b [16, 20], miR-223 [18], miR-545-3p [23] and miR-194-5p [20] were down-regulated in serum of $\mathrm{AD}$ patients compared with that of normal subjects. In addition, serum miR-206 was enhanced in the individuals with amnestic mild cognitive impairment tend to progress to AD [17]. Furthermore, circulating miR-28-3p level was elevated but expressions of miR-125b, miR-9 and miR-191-5p were reduced in the APP/PS1 transgenic mouse model of AD [24]. Next, we detailed introduced several serum miRNAs which were studied more in AD progression.

miR-195 and $A D$

The serum level of miR-195 is elevated in AD patients [15]. Zhang et al reported that decreased expression of mitofusin-2 (mfn2) was linked with mitochondrial dysfunction during the progression of $\mathrm{AD}$, which was considered as $\mathrm{mfn} 2$ level being partly modulated by miR195 [25]. Discordantly, other studies identified miR-195 as a negative modulator in $\mathrm{AD}$ advancement. The formation of $A \beta$ plaques is one of the crucial pathological hallmarks of $\mathrm{AD}$ [26-28]. A $\beta$ peptide is produced from amyloid precursor protein (APP) which is cleavage by beta-site APP cleaving enzyme 1 (BACE1) [26]. The level of BACE1 was reduced by miR-195 via its binding site targeting BACE1 3'UTR, and down-regulation of miR195 resulted amyloidogenesis in senescence-accelerated mice (SAMP8) or in chronic brain hypoperfusion rats with bilateral common carotid artery occlusion [27, 28]. On the basis, abnormal expression level of miR-195 promotes the pathological process of AD.

miR-155 and $A D$

Neuroinflammation and immune-defense are considered as key factors in AD progression and pathogenesis [29].
MiR-155 is one of the most well studied immune-related miRNAs in AD-related neuroinflammatory events. Persistent microglial activation is able to initiate neuronal damage and eventually causes AD [30]. MiR-155 level was remarkably increased in SH-5Y5Y cells transfected with Swedish mutant of $\mathrm{APP}_{695}$ accompanying with higher APP and $A \beta_{1-40}$ production and enhanced inflammatory marker expressions [31]. A highly expression level of miR-155 was also confirmed in $3 \times \mathrm{Tg}$ AD animal model [32]. This early up-regulated miR-155 and c-Jun simultaneously accompanied with an enhanced activation of microglia and astrocyte, thus triggered the production of inflammatory mediators [32]. Moreover, dipeptidyl vinyl sulfone (VS) reduced A $\beta$-induced microglia activation via suppression the expression of inflammatory mediators, as well as prevention the elevated expression levels of miR-155 and miR-146a upon $A \beta$ treatment [30]. miR-155 was also observed differentially expressed in blood-derived monocytes and monocyte-derived macrophages which were isolated from blood of $\mathrm{AD}$, mild cognitive impairment (MCI) patients and healthy controls [33]. Lipopolysaccharide (LPS) treatment can lead to peripheral- and neuro- inflammation [34]. The recipient mice that received serum-derived exosomes from LPS-challenged mice showed characteristics of enhanced microglial activation, elevated pro-inflammation cytokine and its mRNA production, especially increased the miR-155 expression level [35]. In addition, miR- 155 contributed to regulation of AD disease via activation of diverse of $\mathrm{T}$ cells functions during inflammation which may alleviated AD related severe pathologies [36]. On the basis, as a key inflammation and immune related miRNA, miR-155 exerts a positive function in promoting screening effective treatments for $\mathrm{AD}$.

\section{miR-34a and $A D$}

The expression of peripheral miR-34a was dramatically up-regulated in AD subjects compared to normal elderly controls [16, 37]. MiR-34a was also highly expressed in specific brain regions of $\mathrm{AD}$ patients, $3 \times \mathrm{Tg}$ - $\mathrm{AD}$ mouse model as well as cerebral cortex of APPswe/PS mice [3840]. Moreover, the enhanced miR-34a expression in brain compared to age matched healthy control was closely associated with severity of AD pathology [38]. P53 is a crucial response element of miR-34a, p53/miR-34a axis promotes cell apoptosis via activating caspase-3 and suppressing Sirt1 and Bcl2 expressions in AD transgenic mice brain [39, 41]. Another p53-family member Tap73 (p73) drives miR-34a expression through binding specific sites of miR-34a promoter. A remarkable raised miR34a/p73 expression was found in AD hippocampus, which participated in modulating synaptic activity by lessening synaptotagmin-1 expression in brain from AD patients 
[42]. In addition, knockout of miR-34a in APP/PS1 mice decreased $A \beta$ plague production and improved cognitive function by depression of $\gamma$-secretase activity [40]. Thus, miR-34a is considered as a key modulator in process of AD pathology.

miR-9 and $A D$

The declined level of whole-blood has-miR-9-5p was tightly linked with a raised risk of $\mathrm{AD}[24,43]$. In $\mathrm{A} \beta$ treated hippocampal cells, as well as in APP23 transgenic mice or human $\mathrm{AD}$ cortex, miR-9 and miR-181c were down-regulated and exerted their roles in brain homeostasis via targeting TGFBI, TRIM2, SIRT1 and BTBD3 [44]. A $\beta_{42}$ treatment initiated CAMK2-AMPK signaling activation and synaptotoxic impairment as the result of the reduced expression of miR-9, while upregulation of miR-9 was sufficient to restore $A \beta_{42-}$ induced dendritic spine loss [45]. Thus, as a drug treatment target, osthole exerts its powerful neuroprotective effect against AD via promoting miR-9 level, reducing CAMKK2 and p-AMPK $\alpha$ expressions and subsequently suppressing the Notch signaling pathway [46, 47]. Hence miR-9 was a neuroprotective regulator in $\mathrm{AD}$ development.

miR-206 and $A D$

The level of serum miR-206 and miR-132 were elevated in MCI patients compared with age-matched normal controls. Combining detection of miR-206 and miR-132 achieved a highest areas under curves (AUC), which is an index of miRNA's diagnostic performance [48, 49]. Yet, the serum miR-206 level was increased in amnestic MCI (aMCI)-AD patients than aMCI-aMCI group whereas no notable differences in serum levels of miR-132 [17]. The miR-206 levels were also up-regulated in the Tg2576 mice brain and the temporal cortex of human AD brains [50], the hippocampal tissue and plasma of embryonic APP/PS1 transgenic mice [51]. The enhanced expression of miR-206 major promoted the detrimental effect of $A \beta_{42}$ on brain-derived neurotrophic factor (BDNF) via inhibiting the level of BDNF [50, 51]. Further, miR-206 inhibitor is able to relive the detrimental effects of $A \beta_{42}$, and it is a target of donepezil, a drug approved for treating $\mathrm{AD}$ in clinic [52]. Therefore, miR-206 was a modulator to exacerbate the $\mathrm{AD}$ advancement.

miR-125b and $A D$

The serum miR-125b was down-regulated in AD patients compared with that of control subjects [15, 53-55]. In addition, decreased circulating miR-125b was also found in the APP/PS1 transgenic mouse models of AD [24]. In the $A \beta$ pathological condition, the reduced expression of miR-125b is a critical event for the neurotoxic effect in cortical neurons. $17 \beta$-estradiol can protect neurons from the $A \beta$-peptide caused neurotoxicity via up-regulation miR-125b expression [56]. However, it is inconsistently with the protection role of miR-125b in AD. MiR-125b was found highly expression in AD patients [57]. Upregulation of miR-125b caused tau hyperphosphorylation, inhibited cell proliferation, promoted apoptosis, induced inflammation and oxidative stress by activation of CDK5 and p35/25, p44/42-MAPK signaling pathway, and suppressed expressions of forkhead box Q1 (FOXQ1), anti-apoptotic factor Bcl-W, and sphingosine kinase 1 (SphK1) [57-59]. It is probably that the aberrant expression of miR-125b contributes to the neural dysfunction in AD brain.

\section{miR-29 and $A D$}

The serum of miR-29 expression was remarkably depressed in AD patients verse control subjects [15, 6062]. The major events associated with the aberrant expression of miR-29 up-regulated the $A \beta$ precursor protein BACE1 expression and subsequent caused $A \beta$ accumulation [62, 63]. MiR-29c directly targets the 3'UTR of BACE1 mRNA [63]. Overexpression of miR29 was able to reduce the level of BACE1 and A $\beta$ accumulation in vitro, and ameliorate learning and memory in SAMP9 mice partially through enhancing the activity of protein kinase A/cAMP response elementbinding protein [62, 63]. Thus, its highly pure and biologically active pre-miR-29b deliver using polyplexes to $\mathrm{N} 2 \mathrm{a} 695$ cells can reduce BACE1 expression and $\mathrm{A} \beta_{42}$ level, which was considered as a potentially therapy way for $\mathrm{AD}$ [61]. In AD brains, neuron navigator 3 (NAV3) was highly expressed as the result of the attenuated miR$29 \mathrm{a}$ and miR-29c expressions, which also referred to dysregulation of axon guidance $[64,65]$. The up-regulated expression of miR-29c-3p and miR-29b also exhibited neuroprotective functions in $\mathrm{AD}$ via targeting signal activators of transcription 3 (STAT3) and specificity protein 1 (Sp1), respectively [15, 60]. Thus, miR-29 family are potentially biomarkers for $\mathrm{AD}$ treatment.

\section{CSF miRNAs and AD}

Cerebrospinal fluid is a continuum of the brain, which is an attractive source of biomarkers reflecting central neuropathological features of the brain diseases including AD [66]. Recently, emerging studies have suggested that CSF contains circulating miRNAs, which were critical biomarkers with a high predictive accuracy in the pathogenesis process of $\mathrm{AD}$ (Fig. 1). The expression levels of miR-let-7e [67], miR-let-7b [67, 68], miR-29a [69], miR-613 [70], miR-206 [51], miR-222 [71], miR125b [71], miR-let7i-5p [20] and miR-15a-5p [20] were increased, while miR-135a [72], miR-200b [72], miR193b [73], miR-299-5p [74], miR-29c [20, 75], miR-27a- 
3p [76], miR-210 [77], miR-384 [78] and miR-214-3p [79] were decreased in the CSF from AD patients compared to healthy controls. The elevated miR-let-7b level in CSF from AD patients mainly originated from CD4+ $\mathrm{T}$ lymphocyte and was associated with neurotoxicity and t-tau/p-tau expression $[67,68]$. miR613 or miR-206 was responsible for the AD pathology via suppression the neuroprotector-BDNF [51, 70], which also accompanied with a decreased expression of miR-29c [75]. Under AD pathological conditions, the expressions of miR-384, miR-135a and miR-200b in CSF were declined, which attenuated their repression roles on the APP and BACE1 levels. Meanwhile, A $\beta_{42}$ could also restrain miR-384 and miR-200 expression. The above may generated a vicious cycle resulted in accumulation of $\mathrm{A} \beta_{42}$ [72, 78]. Moreover, miR-193b was negatively correlated with $A \beta_{42}$ in the CSF of dementia of Alzheimer-type (DAT) patients [73]. The low level of miR-27a-3p was linked with the enhanced tau but decreased $A \beta$ levels [76] in the CSF from AD patients. The expressions of miR-299-5p and miR-214-3p were reduced upon $\mathrm{AD}$ conditions and resulted in autophagy by disinhibition of Atg5, LC3 3 II and Beclin1 levels, respectively $[73,79]$. MiR-210 expression was abated in the CSF and serum accompanied with the decreased level of VEGF, which were associated with the severity of the AD [77]. With the thorough studies on CSF miRNAs in $\mathrm{AD}$, they will be used as biomarkers to assess disease progression and therapeutic efficacy.

\section{Circulating IncRNAs and Alzheimer's disease}

Recently, IncRNAs are also found in circulating fluid and play pivotal roles in various diseases [80]. LncRNAs are classically defined as > 200 nucleotides transcripts lacking protein-coding ability with biological regulatory and modificatory functions [81, 82]. As advanced transcriptome-wide profiling approach, emerging number of IncRNAs were comprehensively identified dysregulated in AD pathological brains [83, 84]. Circulating lncRNA was also described to participate in the occurrence and development of AD (Fig. 1) [85]. The lncRNA BACE1 level was dramatically up-regulated in AD patient's plasma compared with normal control subjects, while no significantly alteration of plasma lncRNA 17A, 51A and BC200. Accordantly, Manzine et $a l$ also confirmed that plasma BACE1 level was elevated in AD patients [86]. However, Marison et al proved that there was no dramatically difference of BACE1 expression in blood between control, $\mathrm{AD}$ and non-AD neuropathology's individuals. Interestingly, it was still considered as a potential marker used in diagnosis as that have somehow been involved with $\mathrm{AD}$ or $\mathrm{AD}$-related elements [87]. In the context, we have introduced that BACE1 is a key modulator and its mediated the production of $A \beta$ from APP is the rate limiting step in AD progression. BACE1 is also positively regulated by its antisense transcript (lncRNA BACE1-AS) [88], which partly binds with BACE1 mRNA and promotes BACE1 expression [89]. LncRNA BACE1 and BACE1-AS were checked highly expressed in the blood in brain related disease [90]. They promoted $\mathrm{AD}$ pathogenesis via enhancing $A \beta$ and APP level and they were able to participate in learning and memory impairment via being stabilized by the primarily neuronal RNA-binding protein $\mathrm{HuD}$ in $\mathrm{AD}$ advancement [89]. In addition, the abnormal expressed BACE1 can be not only a biomarker for AD diagnosis, but also a therapeutic target by systemic delivery of BACE1 siRNA and its inhibitors [91, 92]. In brief, the lncRNAs in circulatory fluid from AD patients need to be intensively studied for supplying novel biomarkers or providing new drug treatment targets in $\mathrm{AD}$ development in the future.

\section{Conclusions}

The dementia related diseases are difficult to be definitive diagnosed. They are traditionally based on the history of the disease, the pattern of cognitive impairment, and on additional parameters evaluated via clinical examination, including blood tests and brain structural imaging, to exclude nondegenerative causes of the symptoms [93]. It is necessary and urgent to seek novel simple and effective biomarkers for diagnosis specific forms of dementia earlier, also in the pre-dementia stages of the disease, and with more specificity. As the increasing number of circulating miRNAs and lncRNAs are being to be reported abnormally expressed in $\mathrm{AD}$ patients. Characterizations of these AD-associated circulating miRNAs and lncRNAs offer the possibility of providing new insights into disease pathogenesis. In consideration of that non-coding RNAs in circulatory fluids are easily accessible and relatively stable, it is more reality to make a correct clinical prognosis for AD. Thus, confirming the aberrant expressed circulating miRNAs and lncRNAs will provide an effective testing tools for treatment of $\mathrm{AD}$ in the future.

\section{References}

[1] Reitz C, Mayeux R (2014). Alzheimer disease: epidemiology, diagnostic criteria, risk factors and biomarkers. Biochem Pharmacol, 88:640-651.

[2] Sumner IL, Edwards RA, Asuni AA, Teeling JL (2018). Antibody Engineering for Optimized Immunotherapy in Alzheimer's Disease. Front Neurosci, 12:254.

[3] Jarrell JT, Gao L, Cohen DS, Huang X (2018). Network Medicine for Alzheimer's Disease and Traditional Chinese Medicine. Molecules, 23.

[4] Magalingam KB, Radhakrishnan A, Ping NS, Haleagrahara N (2018). Current Concepts of Neurodegenerative Mechanisms in Alzheimer's 
Disease. Biomed Res Int, 2018:3740461.

[5] Kumar A, Singh A, Ekavali (2015). A review on Alzheimer's disease pathophysiology and its management: an update. Pharmacol Rep, 67:195-203.

[6] Livingston G, Sommerlad A, Orgeta V, Costafreda SG, Huntley J, Ames D, et al. (2017). Dementia prevention, intervention, and care. Lancet, 390:2673-2734.

[7] Prince MJ, Wu F, Guo Y, Gutierrez Robledo LM, O'Donnell M, Sullivan R, et al. (2015). The burden of disease in older people and implications for health policy and practice. Lancet, 385:549-562.

[8] Bartel DP (2004). MicroRNAs: genomics, biogenesis, mechanism, and function. Cell, 116:281-297.

[9] Tan L, Yu JT, Tan L (2015). Causes and Consequences of MicroRNA Dysregulation in Neurodegenerative Diseases. Mol Neurobiol, 51:1249-1262.

[10] Baldassarre A, Felli C, Prantera G, Masotti A (2017). Circulating microRNAs and Bioinformatics Tools to Discover Novel Diagnostic Biomarkers of Pediatric Diseases. Genes (Basel), 8.

[11] Weber JA, Baxter DH, Zhang S, Huang DY, Huang $\mathrm{KH}$, Lee MJ, et al. (2010). The microRNA spectrum in 12 body fluids. Clin Chem, 56:1733-1741.

[12] Chen X, Ba Y, Ma L, Cai X, Yin Y, Wang K, et al. (2008). Characterization of microRNAs in serum: a novel class of biomarkers for diagnosis of cancer and other diseases. Cell Res, 18:997-1006.

[13] Yang TT, Liu CG, Gao SC, Zhang Y, Wang PC (2018). The Serum Exosome Derived MicroRNA-135a, -193b, and -384 Were Potential Alzheimer's Disease Biomarkers. Biomed Environ Sci, 31:87-96.

[14] Kumar S, Reddy PH (2018). MicroRNA-455-3p as a Potential Biomarker for Alzheimer's Disease: An Update. Front Aging Neurosci, 10:41.

[15] Wu Y, Xu J, Xu J, Cheng J, Jiao D, Zhou C, et al. (2017). Lower Serum Levels of miR-29c-3p and miR$19 b-3 p$ as Biomarkers for Alzheimer's Disease. Tohoku J Exp Med, 242:129-136.

[16] Chen J, Qi Y, Liu CF, Lu JM, Shi J, Shi Y (2018). MicroRNA expression data analysis to identify key miRNAs associated with Alzheimer's disease. J Gene Med, 20:e3014.

[17] Xie B, Liu Z, Jiang L, Liu W, Song M, Zhang Q, et al. (2017). Increased Serum miR-206 Level Predicts Conversion from Amnestic Mild Cognitive Impairment to Alzheimer's Disease: A 5-Year Followup Study. J Alzheimers Dis, 55:509-520.

[18] Jia LH, Liu YN (2016). Downregulated serum miR223 servers as biomarker in Alzheimer's disease. Cell Biochem Funct, 34:233-237.

[19] Wu Q, Ye X, Xiong Y, Zhu H, Miao J, Zhang W, et al. (2016). The Protective Role of microRNA-200c in Alzheimer's Disease Pathologies Is Induced by Beta Amyloid-Triggered Endoplasmic Reticulum Stress. Front Mol Neurosci, 9:140.

[20] Sorensen SS, Nygaard AB, Christensen T (2016). miRNA expression profiles in cerebrospinal fluid and blood of patients with Alzheimer's disease and other types of dementia - an exploratory study. Transl Neurodegener, 5:6.
Hara N, Kikuchi M, Miyashita A, Hatsuta H, Saito Y, Kasuga K, et al. (2017). Serum microRNA miR-501$3 p$ as a potential biomarker related to the progression of Alzheimer's disease. Acta Neuropathol Commun, 5:10.

[22] Zhang Y, Xing H, Guo S, Zheng Z, Wang H, Xu D (2016). MicroRNA-135b has a neuroprotective role via targeting of beta-site APP-cleaving enzyme 1. Exp Ther Med, 12:809-814.

[23] Cosin-Tomas M, Antonell A, Llado A, Alcolea D, Fortea J, Ezquerra M, et al. (2017). Plasma miR-34a$5 p$ and miR-545-3p as Early Biomarkers of Alzheimer's Disease: Potential and Limitations. Mol Neurobiol, 54:5550-5562.

[24] Hong H, Li Y, Su B (2017). Identification of Circulating miR-125b as a Potential Biomarker of Alzheimer's Disease in APP/PS1 Transgenic Mouse. J Alzheimers Dis, 59:1449-1458.

[25] Zhang R, Zhou H, Jiang L, Mao Y, Cui X, Xie B, et al. (2016). MiR-195 dependent roles of mitofusin2 in the mitochondrial dysfunction of hippocampal neurons in SAMP8 mice. Brain Res, 1652:135-143.

[26] Vetrivel KS, Barman A, Chen Y, Nguyen PD, Wagner SL, Prabhakar R, et al. (2011). Loss of cleavage at beta'-site contributes to apparent increase in betaamyloid peptide (Abeta) secretion by beta-secretase (BACE1)-glycosylphosphatidylinositol (GPI) processing of amyloid precursor protein. J Biol Chem, 286:26166-26177.

[27] Zhu HC, Wang LM, Wang M, Song B, Tan S, Teng JF, et al. (2012). MicroRNA-195 downregulates Alzheimer's disease amyloid-beta production by targeting BACE1. Brain Res Bull, 88:596-601.

[28] Sun LH, Ban T, Liu CD, Chen QX, Wang X, Yan ML, et al. (2015). Activation of $\mathrm{Cdk} 5 / \mathrm{p} 25$ and tau phosphorylation following chronic brain hypoperfusion in rats involves microRNA-195 downregulation. J Neurochem, 134:1139-1151.

Rubio-Perez JM, Morillas-Ruiz JM (2012). A review: inflammatory process in Alzheimer's disease, role of cytokines. ScientificWorldJournal, 2012:756357.

[30] Falcao AS, Carvalho LA, Lidonio G, Vaz AR, Lucas SD, Moreira R, et al. (2017). Dipeptidyl Vinyl Sulfone as a Novel Chemical Tool to Inhibit HMGB1/NLRP3Inflammasome and Inflamma-miRs in AbetaMediated Microglial Inflammation. ACS Chem Neurosci, 8:89-99.

[31] Fernandes A, Ribeiro AR, Monteiro M, Garcia G, Vaz AR, Brites D (2018). Secretome from SH-SY5Y APPSwe cells trigger time-dependent CHME3 microglia activation phenotypes, ultimately leading to miR-21 exosome shuttling. Biochimie.

[32] Guedes JR, Custodia CM, Silva RJ, de Almeida LP, Pedroso de Lima MC, Cardoso AL (2014). Early miR155 upregulation contributes to neuroinflammation in Alzheimer's disease triple transgenic mouse model. Hum Mol Genet, 23:6286-6301.

[33] Guedes JR, Santana I, Cunha C, Duro D, Almeida MR, Cardoso AM, et al. (2016). MicroRNA deregulation and chemotaxis and phagocytosis impairment in 
Alzheimer's disease. Alzheimers Dement (Amst), 3:717.

[34] Henry CJ, Huang Y, Wynne AM, Godbout JP (2009). Peripheral lipopolysaccharide (LPS) challenge promotes microglial hyperactivity in aged mice that is associated with exaggerated induction of both proinflammatory IL-1beta and anti-inflammatory IL-10 cytokines. Brain Behav Immun, 23:309-317.

[35] Li JJ, Wang B, Kodali MC, Chen C, Kim E, Patters BJ, et al. (2018). In vivo evidence for the contribution of peripheral circulating inflammatory exosomes to neuroinflammation. J Neuroinflammation, 15:8.

[36] Song J, Lee JE (2015). miR-155 is involved in Alzheimer's disease by regulating $\mathrm{T}$ lymphocyte function. Front Aging Neurosci, 7:61.

[37] Schipper HM, Maes OC, Chertkow HM, Wang E (2007). MicroRNA expression in Alzheimer blood mononuclear cells. Gene Regul Syst Bio, 1:263-274.

[38] Sarkar S, Jun S, Rellick S, Quintana DD, Cavendish JZ, Simpkins JW (2016). Expression of microRNA34a in Alzheimer's disease brain targets genes linked to synaptic plasticity, energy metabolism, and resting state network activity. Brain Res, 1646:139-151.

[39] Wang X, Liu P, Zhu H, Xu Y, Ma C, Dai X, et al. (2009). miR-34a, a microRNA up-regulated in a double transgenic mouse model of Alzheimer's disease, inhibits bcl2 translation. Brain Res Bull, 80:268-273.

[40] Jian C, Lu M, Zhang Z, Liu L, Li X, Huang F, et al. (2017). miR-34a knockout attenuates cognitive deficits in APP/PS1 mice through inhibition of the amyloidogenic processing of APP. Life Sci, 182:104111.

[41] Li LH, Tu QY, Deng XH, Xia J, Hou DR, Guo K, et al. (2017). Mutant presenilin2 promotes apoptosis through the $\mathrm{p} 53 / \mathrm{miR}-34 \mathrm{a}$ axis in neuronal cells. Brain Res, 1662:57-64.

[42] Agostini M, Tucci P, Killick R, Candi E, Sayan BS, Rivetti di Val Cervo P, et al. (2011). Neuronal differentiation by TAp73 is mediated by microRNA34 a regulation of synaptic protein targets. Proc Natl Acad Sci U S A, 108:21093-21098.

[43] Yilmaz SG, Erdal ME, Ozge AA, Sungur MA (2016). Can Peripheral MicroRNA Expression Data Serve as Epigenomic (Upstream) Biomarkers of Alzheimer's Disease? OMICS, 20:456-461.

[44] Schonrock N, Humphreys DT, Preiss T, Gotz J (2012). Target gene repression mediated by miRNAs miR181c and miR-9 both of which are down-regulated by amyloid-beta. J Mol Neurosci, 46:324-335.

[45] Chang F, Zhang LH, Xu WP, Jing P, Zhan PY (2014). microRNA-9 attenuates amyloidbeta-induced synaptotoxicity by targeting calcium/calmodulindependent protein kinase kinase 2. Mol Med Rep, 9:1917-1922.

[46] Li S, Yan Y, Jiao Y, Gao Z, Xia Y, Kong L, et al. (2016). Neuroprotective Effect of Osthole on Neuron Synapses in an Alzheimer's Disease Cell Model via Upregulation of MicroRNA-9. J Mol Neurosci, 60:7181.

[47] Li SH, Gao P, Wang LT, Yan YH, Xia Y, Song J, et al.
(2017). Osthole Stimulated Neural Stem Cells Differentiation into Neurons in an Alzheimer's Disease Cell Model via Upregulation of MicroRNA-9 and Rescued the Functional Impairment of Hippocampal Neurons in APP/PS1 Transgenic Mice. Front Neurosci, 11:340.

[48] Xie B, Zhou H, Zhang R, Song M, Yu L, Wang L, et al. (2015). Serum miR-206 and miR-132 as Potential Circulating Biomarkers for Mild Cognitive Impairment. J Alzheimers Dis, 45:721-731.

[49] Moon J, Lee ST, Kong IG, Byun JI, Sunwoo JS, Shin JW, et al. (2016). Early diagnosis of Alzheimer's disease from elevated olfactory mucosal miR-206 level. Sci Rep, 6:20364.

[50] Lee ST, Chu K, Jung KH, Kim JH, Huh JY, Yoon H, et al. (2012). miR-206 regulates brain-derived neurotrophic factor in Alzheimer disease model. Ann Neurol, 72:269-277.

[51] Tian N, Cao Z, Zhang Y (2014). MiR-206 decreases brain-derived neurotrophic factor levels in a transgenic mouse model of Alzheimer's disease. Neurosci Bull, 30:191-197.

[52] Wang CN, Wang YJ, Wang H, Song L, Chen Y, Wang JL, et al. (2017). The Anti-dementia Effects of Donepezil Involve miR-206-3p in the Hippocampus and Cortex. Biol Pharm Bull, 40:465-472.

[53] Galimberti D, Villa C, Fenoglio C, Serpente M, Ghezzi L, Cioffi SM, et al. (2014). Circulating miRNAs as potential biomarkers in Alzheimer's disease. J Alzheimers Dis, 42:1261-1267.

[54] Tan L, Yu JT, Liu QY, Tan MS, Zhang W, Hu N, et al. (2014). Circulating miR-125b as a biomarker of Alzheimer's disease. J Neurol Sci, 336:52-56.

[55] Lugli G, Cohen AM, Bennett DA, Shah RC, Fields CJ, Hernandez AG, et al. (2015). Plasma Exosomal miRNAs in Persons with and without Alzheimer Disease: Altered Expression and Prospects for Biomarkers. PLoS One, 10:e0139233.

[56] Micheli F, Palermo R, Talora C, Ferretti E, Vacca A, Napolitano M (2016). Regulation of proapoptotic proteins Bak1 and $\mathrm{p} 53$ by miR-125b in an experimental model of Alzheimer's disease: Protective role of 17beta-estradiol. Neurosci Lett, 629:234-240.

[57] Ma X, Liu L, Meng J (2017). MicroRNA-125b promotes neurons cell apoptosis and Tau phosphorylation in Alzheimer's disease. Neurosci Lett, 661:57-62.

[58] Banzhaf-Strathmann J, Benito E, May S, Arzberger T, Tahirovic S, Kretzschmar H, et al. (2014). MicroRNA$125 \mathrm{~b}$ induces tau hyperphosphorylation and cognitive deficits in Alzheimer's disease. EMBO J, 33:16671680.

[59] Jin Y, Tu Q, Liu M (2018). MicroRNA125b regulates Alzheimer's disease through SphK1 regulation. Mol Med Rep, 18:2373-2380.

[60] Villa C, Ridolfi E, Fenoglio C, Ghezzi L, Vimercati R, Clerici F, et al. (2013). Expression of the transcription factor Sp1 and its regulatory hsa-miR-29b in peripheral blood mononuclear cells from patients with Alzheimer's disease. J Alzheimers Dis, 35:487-494. 
[61] Pereira PA, Tomas JF, Queiroz JA, Figueiras AR, Sousa F (2016). Recombinant pre-miR-29b for Alzheimer s disease therapeutics. Sci Rep, 6:19946.

[62] Yang G, Song Y, Zhou X, Deng Y, Liu T, Weng G, et al. (2015). MicroRNA-29c targets beta-site amyloid precursor protein-cleaving enzyme 1 and has a neuroprotective role in vitro and in vivo. Mol Med Rep, 12:3081-3088.

[63] Lei X, Lei L, Zhang Z, Zhang Z, Cheng Y (2015). Downregulated miR-29c correlates with increased BACE1 expression in sporadic Alzheimer's disease. Int J Clin Exp Pathol, 8:1565-1574.

[64] Shioya M, Obayashi S, Tabunoki H, Arima K, Saito Y, Ishida T, et al. (2010). Aberrant microRNA expression in the brains of neurodegenerative diseases: miR-29a decreased in Alzheimer disease brains targets neurone navigator 3. Neuropathol Appl Neurobiol, 36:320-330.

[65] Zong Y, Yu P, Cheng H, Wang H, Wang X, Liang C, et al. (2015). miR-29c regulates NAV3 protein expression in a transgenic mouse model of Alzheimer's disease. Brain Res, 1624:95-102.

[66] Ghidoni R, Benussi L, Paterlini A, Albertini V, Binetti G, Emanuele E (2011). Cerebrospinal fluid biomarkers for Alzheimer's disease: the present and the future. Neurodegener Dis, 8:413-420.

[67] Derkow K, Rossling R, Schipke C, Kruger C, Bauer J, Fahling M, et al. (2018). Distinct expression of the neurotoxic microRNA family let-7 in the cerebrospinal fluid of patients with Alzheimer's disease. PLoS One, 13:e0200602.

[68] Liu Y, He X, Li Y, Wang T (2018). Cerebrospinal fluid CD4+ $\mathrm{T}$ lymphocyte-derived miRNA-let-7b can enhances the diagnostic performance of Alzheimer's disease biomarkers. Biochem Biophys Res Commun, 495:1144-1150.

[69] Muller M, Jakel L, Bruinsma IB, Claassen JA, Kuiperij HB, Verbeek MM (2016). MicroRNA-29a Is a Candidate Biomarker for Alzheimer's Disease in Cell-Free Cerebrospinal Fluid. Mol Neurobiol, 53:2894-2899.

[70] Li W, Li X, Xin X, Kan PC, Yan Y (2016). MicroRNA613 regulates the expression of brain-derived neurotrophic factor in Alzheimer's disease. Biosci Trends, 10:372-377.

[71] Dangla-Valls A, Molinuevo JL, Altirriba J, SanchezValle R, Alcolea D, Fortea J, et al. (2017). CSF microRNA Profiling in Alzheimer's Disease: a Screening and Validation Study. Mol Neurobiol, 54:6647-6654.

[72] Liu CG, Wang JL, Li L, Xue LX, Zhang YQ, Wang PC (2014). MicroRNA-135a and -200b, potential Biomarkers for Alzheimers disease, regulate beta secretase and amyloid precursor protein. Brain Res, 1583:55-64.

[73] Liu CG, Song J, Zhang YQ, Wang PC (2014). MicroRNA-193b is a regulator of amyloid precursor protein in the blood and cerebrospinal fluid derived exosomal microRNA-193b is a biomarker of Alzheimer's disease. Mol Med Rep, 10:2395-2400.

[74] Zhang Y, Liu C, Wang J, Li Q, Ping H, Gao S, et al.
(2016). MiR-299-5p regulates apoptosis through autophagy in neurons and ameliorates cognitive capacity in APPswe/PS1dE9 mice. Sci Rep, 6:24566. Yang G, Song Y, Zhou X, Deng Y, Liu T, Weng G, et al. (2015). DNA methyltransferase 3, a target of microRNA-29c, contributes to neuronal proliferation by regulating the expression of brain-derived neurotrophic factor. Mol Med Rep, 12:1435-1442.

[76] Sala Frigerio C, Lau P, Salta E, Tournoy J, Bossers K, Vandenberghe R, et al. (2013). Reduced expression of hsa-miR-27a-3p in CSF of patients with Alzheimer disease. Neurology, 81:2103-2106.

[77] Zhu Y, Li C, Sun A, Wang Y, Zhou S (2015). Quantification of microRNA-210 in the cerebrospinal fluid and serum: Implications for Alzheimer's disease. Exp Ther Med, 9:1013-1017.

[78] Liu CG, Wang JL, Li L, Wang PC (2014). MicroRNA384 regulates both amyloid precursor protein and betasecretase expression and is a potential biomarker for Alzheimer's disease. Int J Mol Med, 34:160-166.

[79] Zhang Y, Li Q, Liu C, Gao S, Ping H, Wang J, et al. (2016). MiR-214-3p attenuates cognition defects via the inhibition of autophagy in SAMP8 mouse model of sporadic Alzheimer's disease. Neurotoxicology, 56:139-149.

[80] Jiang X, Lei R, Ning Q (2016). Circulating long noncoding RNAs as novel biomarkers of human diseases. Biomark Med, 10:757-769.

[81] Lee C, Kikyo N (2012). Strategies to identify long noncoding RNAs involved in gene regulation. Cell Biosci, 2:37.

[82] Ponting CP, Oliver PL, Reik W (2009). Evolution and functions of long noncoding RNAs. Cell, 136:629-641.

[83] Lee DY, Moon J, Lee ST, Jung KH, Park DK, Yoo JS, et al. (2015). Distinct Expression of Long Non-Coding RNAs in an Alzheimer's Disease Model. J Alzheimers Dis, $45: 837-849$.

[84] Fang M, Zhang $P$, Zhao Y, Liu X (2017). Bioinformatics and co-expression network analysis of differentially expressed lncRNAs and mRNAs in hippocampus of APP/PS1 transgenic mice with Alzheimer disease. Am J Transl Res, 9:1381-1391.

[85] Feng L, Liao YT, He JC, Xie CL, Chen SY, Fan HH, et al. (2018). Plasma long non-coding RNA BACE1 as a novel biomarker for diagnosis of Alzheimer disease. BMC Neurol, 18:4.

[86] Manzine PR, Souza MDS, Cominetti MR (2016). BACE1 levels are increased in plasma of Alzheimer's disease patients compared with matched cognitively healthy controls. Per Med, 13:531-540.

[87] Herrera-Rivero $M$, Elena Hernandez-Aguilar $M$, Emiliano Aranda-Abreu G (2015). A strategy focused on MAPT, APP, NCSTN and BACE1 to build blood classifiers for Alzheimer's disease. J Theor Biol, 376:32-38.

[88] Faghihi MA, Modarresi F, Khalil AM, Wood DE, Sahagan BG, Morgan TE, et al. (2008). Expression of a noncoding RNA is elevated in Alzheimer's disease and drives rapid feed-forward regulation of betasecretase. Nat Med, 14:723-730. 
[89] Kang MJ, Abdelmohsen K, Hutchison ER, Mitchell SJ, Grammatikakis I, Guo R, et al. (2014). HuD regulates coding and noncoding RNA to induce APP-->Abeta processing. Cell Rep, 7:1401-1409.

[90] Mazdeh M, Komaki A, Omrani MD, Gharzi V, Sayad A, Taheri M, et al. (2018). Expression analysis of betasecretase 1 (BACE1) and its naturally occurring antisense (BACE1-AS) in blood of epileptic patients. Neurol Sci, 39:1565-1569.

[91] Wang P, Zheng X, Guo Q, Yang P, Pang X, Qian K, et al. (2018). Systemic delivery of BACE1 siRNA through neuron-targeted nanocomplexes for treatment of Alzheimer's disease. J Control Release, 279:220233.

[92] Sakamoto K, Matsuki S, Matsuguma K, Yoshihara T, Uchida N, Azuma F, et al. (2017). BACE1 Inhibitor Lanabecestat (AZD3293) in a Phase 1 Study of Healthy Japanese Subjects: Pharmacokinetics and Effects on Plasma and Cerebrospinal Fluid Abeta Peptides. J Clin Pharmacol, 57:1460-1471.

[93] Lashley T, Schott JM, Weston P, Murray CE, Wellington H, Keshavan A, et al. (2018). Molecular biomarkers of Alzheimer's disease: progress and prospects. Dis Model Mech, 11. 